\title{
Research on Personalized Blog Customization System Based on AJAX
}

\author{
Liu Hongqing ${ }^{1}$, Liu Yan ${ }^{2}$ \\ ${ }^{1}$ Hunan Vocational College of Modern Logistics, Changsha, Hunan, 410131, China \\ ${ }^{2}$ Hunan Mechanical \& Electrical Polytechnic, Changsha, Hunan,410151, China
}

Keywords: AJAX, micro-blog, Web2.0, user experience

\begin{abstract}
In recent years, Java language has developed quite rapidly, and J2EE technology has matured. Many enterprises have been developing projects with J2EE. With the popularity of Web2.0, AJAX technology has gradually developed its powerful function. The introduction of AJAX technology has provided users with better experience and greatly reduced the waiting time of users. When users use the system, they can customize each module according to its own wishes, and can drag the form in the page to the position that you want to put. The system saves each setting of the user to the database, so that the user can see the last setting in the next time when logging into the system. Because the system is integrated with AJAX technology, users can operate this system more conveniently.
\end{abstract}

\section{Introduction}

In recent years, the development of Java language has been very rapid, of which J2EE (Java 2 Platform, Enterprise Edition, Java2 Platform Enterprise Edition) technology has matured, and many enterprises have been using J2EE for project development. With the popularity of Web2.0, AJAX (Asynchronous JavaScript and XML, asynchronous JavaScript and XML) technology has gradually played its role. Though AJAX technology is becoming more and more mature, there are not many AJAX applications. So it is very important to apply AJAX technology to practice.

\section{Tool Introduction}

$\mathrm{J} 2 \mathrm{EE}$ is a set of technical architecture that is completely different from the traditional application development. It can simplify and standardize the development and deployment of the application system, and then improve the portability, security and reuse value.

The J2EE core is a set of technical specifications and guidelines, including all kinds of components, service architecture and technical levels that have common standards and specifications, so that there are good compatibility between different platforms that follow the J2EE architecture, and the incompatibility of information products used in the back end of the past enterprise will be incompatible with each other, leading to the enterprise A dilemma that is difficult to communicate internally or externally.

J2EE includes JSP (Java Server Pages), Servlet, EJB (Enterprise JavaBeans, enterprise JavaBeans), and JMS (JavaBeans). J2EE provides a container platform for multi-layer Web application system. The J2EE application framework enables the same program components to implement different functions according to their deployment in a Web program.

The core specification of J2EE is Enterprise Java Beans (EJB). According to the different characteristics, EJB is currently divided into three kinds: Session Bean, Entity Bean and Message Driven Bean. In which Session Bean and Entity Bean are the earliest used EJB, the two EJB specifications already exist when the EJB 1.x version is released, and Message Driven Bean appears in the specifications of the EJB 2.

J2EE provides an enterprise computing model and running environment for developing and deploying multi tier architecture applications. It provides high availability, security, scalability, and reliability by providing the various services necessary for the enterprise computing environment, making the multi-layer applications deployed on the J2EE platform. Its superiority is that the 
computing platform supports the Java language so that applications developed on J2EE standards can be transplanted across the platform; the Java language is very secure and strict, which enables developers to write very reliable code; J2EE provides the services needed in the enterprise computing, and is more easily used; most of the labels in J2EE All interfaces are defined, such as JNDI (Java Naming and Directory Interface), JDBC, Java Mail, etc., so they can be combined with many manufacturers' products and are easily supported. J2EE has set up a wide and universal standard, which greatly simplifies the application development and migration process.

Eclipse is an extensible open source code IDE (Integrated Development Environment). In November 2001, IBM donated \$40 million worth of source code to form the Eclipse alliance, which is responsible for the subsequent development of this tool. The integrated development environment (IDE) often limits its application scope to the cycle of development, construction and debugging. In order to help the integrated development environment (IDE) overcome the current limitations, the Eclipse platform was created by industry manufacturers. Eclipse allows the integration of tools from different vendors in the same IDE and implements interoperability between tools, which dramatically changes the project workflow and enables developers to focus on the actual embedded target [1].

\section{System Design}

To provide flexible functional modules to meet different needs, and the way of implementation is simple and effective.

(1) Advanced nature

The design scheme is based on advanced technology, using the AJAX technology just beginning to be popular, and using the Hibernate framework for system development.

(2) Extensibility

The original purpose of Java technology is to achieve platform independence. After years of improvement, the features of Java technology "compile once and run everywhere" make the program written with it can be transplanted to any system that has already installed the Java virtual machine.

(3) Normalization

Normalization and standardization are the foundation of a successful system, and also the fundamental guarantee for compatibility and further expansion of the system with other systems. Many key functions of the system exist in the form of modules. Each module accords with the standards and specifications of software system, and is easy to transplant and upgrade.

(4) The principle of interface design

The interface should be friendly, and try to achieve the desktop function that WINDOWS can achieve.

(5) Flexibility

Before starting to develop a system, feasibility study is a necessary step. The purpose of the feasibility study is not to solve the problem, but to determine whether the problem is worth solving, and to assess the risk of an enterprise to determine the probability that the project will be profitable for the enterprise. Otherwise, the development of the system may lose money or even drag down the whole enterprise. So it is necessary to analyze the feasibility of the system before the establishment of the project, to determine whether the original system goal and scale can be realized, and whether the economic benefits that the system can bring after the completion of the system will be worthy of the system.

1) The menu bar above the page is mainly used to set up the "small window" that needs to be displayed in the page, and save the settings state to the database.

2) The module setting will pop up all the modules that can be set up, and click the module that you want to set up, and the corresponding module appears in the page, and the setting option of the menu bar is displayed as a non - setting state.

3) The page body is used to display multiple small windows.

4) "Small window" can be dragged in any page. 
5) The data displayed in "small window" can be read from database dynamically.

6) "small window" can set up the data that you want to add and save it to the database.

7) Click on the small box on the upper right corner of the "small window" to close the "small window", and the corresponding small window menu is displayed as a set state.

8) After the user dragged the "small window", the database saved the current settings, and the status of dragging was displayed in the page.

This system applies Windows XP operation system, adopts the J2EE development kit of Sun company, uses Eclipse as the development platform of the system, and applies Hibernat framework to develop. This system adopts the Oracle database and the Hibernate framework used in database operation.

The main functions of this system are as follows: dragging the mutual position between each module, without refreshing the content, each small module can achieve a personalized custom function, module closing function and module adding function. The system functions are as follows:

(a) Drag: each module can drag each other's relative seats.

(b) Content updating: updating pages can be done without refreshing pages.

(c) Customization: each module has realized a specific customization function.

(d) Shut down: the closure of modules is the concealment of modules.

(e) Add: in the menu bar, click the module you want to add, the module displays the module in the page.

The design of system interface follows the principles of encapsulation, consistency, flexibility, readability and graphic interaction. The friendly design of the interface according to the function of the system.

\section{System Implementation}

The whole system is composed of more than ten small modules, and it is divided into three columns in the page. Each module realizes a specific personalized custom function. The user can not only adjust the overall display mode of the page according to its own needs, but also close some modules or add some modules in the page. Due to the lack of time, the system still has some functions that have not been perfected. Enter the web address in the IE browser, and the system will start running, so that we can enter the main interface of the system. At the top of the page is the menu bar, which displays the menu that can be operated. The main content of the page is to display each module, and each module can drag them to the corresponding location with the mouse.

\section{Summary}

This system combines J2EE and AJAX technology, uses Eclipse as the development tool, realizes the personalized custom function of the system, and can drag the relative position between each module. During the entire graduation project, I learned a lot of new J2EE technologies, had a further understanding of AJAX technology, and learned how to use the Hibernate framework for project development. While completing the system, I also have a new understanding of JavaScript. JavaScript plays a powerful role in page specific effects.

\section{References}

[1] Fernandes, S.L., Gurupur, V.P., Sunder, N.R., Arunkumar, N., Kadry, S.A novel nonintrusive decision support approach for heart rate measurement (2017) Pattern Recognition Letters. https://doi.org/10.1016/j.patrec.2017.07.002

[2] Arunkumar, N., Ramkumar, K., Venkatraman, V., Abdulhay, E., Fernandes, S. L., Kadry, S., \& Segal, S. (2017). Classification of focal and non focal EEG using entropies. Pattern Recognition Letters, vol. 94, pp. 112-117 
[3] Jennifer W. Chan, Yingyue Zhang, and Kathryn E. Uhrich, Amphiphilic Macromolecule Self-Assembled Monolayers Suppress Smooth Muscle Cell Proliferation, Bioconjugate Chemistry, 2015, 26(7), 1359-1369.

[4] Malarkodi, M.P., Arunkumar, N., Venkataraman, V. Gabor wavelet based approach for face recognition (2013) International Journal of Applied Engineering Research, 8 (15), pp. 1831-1840.

[5] Stephygraph, L.R., Arunkumar, N.Brain-actuated wireless mobile robot control through an adaptive human-machine interface (2016) Advances in Intelligent Systems and Computing, 397, pp. 537-549. 